Proceedings of the 12th International Conference "Ion Implantation and Other Applications of Ions and Electrons", ION 2018

\title{
Development of Positron Annihilation Spectroscopy at Joint Institute for Nuclear Research
}

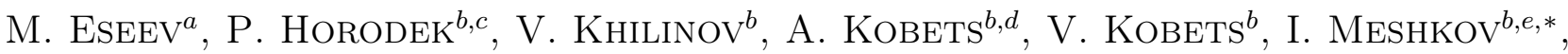 \\ O. ORLOV ${ }^{b}$, K. SiemeK ${ }^{b}$ AND A. A. Sidorin ${ }^{b}$ \\ ${ }^{a}$ Northern Arctic Federal University, 163002 Severnaya Dvina Emb. 17, Arkhangelsk, Russia \\ ${ }^{b}$ Joint Institute for Nuclear Research, Dubna, Russia \\ ${ }^{c}$ Institute of Nuclear Physics Polish Academy of Sciences, PL-31342 Krakow, Poland \\ ${ }^{d}$ Institute of Electrophysics and Radiation Technology of NAS of Ukraine, Kharkov, Ukraine \\ ${ }^{e}$ St. Petersburg State University, St. Petersburg, Russia
}

\begin{abstract}
The method of Positron Annihilation Spectroscopy (PAS) has been under development and application since 2013 at the Low Energy Particle Toroidal Accumulator (LEPTA) facility in the Dzhelepov Laboratory of Nuclear Problems of JINR. The PAS at the LEPTA is based on the slow positron injector of original design that provides a positron flux of a high monochromaticity. The FWHM of positron energy at the exit of the positron source is of $1.5-3 \mathrm{eV}$. The positron energy on the target can be varied in the range $50 \mathrm{eV}-36 \mathrm{keV}(90 \mathrm{keV}$ in the nearest future) with an accuracy of not less than $1 \%$. The first version of a special channel for transportation of the positrons from the source to the target was constructed in 2013. Presently the research is carried out applying the method of the Doppler Broadening of Annihilation Line. In 2018 the channel was developed for application of Positron Annihilation Lifetime Spectroscopy. It will be equipped with instrumentation according to original scheme presented in this article. This article describes three versions of PAS, their peculiarities and specific scheme as well as the parameters of the experimental equipment for PAS used and developed at LEPTA.
\end{abstract}

DOI: 10.12693/APhysPolA.136.314

PACS/topics: 29.27.Fh, 29.27.-h, 61.72.Ff

\section{Introduction}

The method of positron annihilation spectroscopy (PAS) is sensitive to point defects in a solid material and is well-known and used in the material science. It allows to study the concentration of defects with the dimensions smaller than $10 \mathrm{~nm}$ at different depths depending on the positrons' energy [1]. In JINR this method has been under development and application since 2013 when the first experimental station for PAS was founded at the LEPTA facility in Dzhelepov Laboratory of Nuclear Problems of JINR.

The PAS at the LEPTA is based on the slow positron injector that has two unique features:

- high monochromaticity of a positron flux the FWHM of positron energy spectrum at the exit of the positron source is $1.5-3 \mathrm{eV}$;

- positron energy can be varied in the range $50 \mathrm{eV}-36 \mathrm{keV}$ (90 keV in the nearest future) with an accuracy of not less than $1 \%$ by applying a low positive voltage to the source and a negative voltage to the target.

The construction of the slow positron injector was completed in mid-2011, and the group focused on the development of the PAS method. These works were carried

\footnotetext{
*corresponding author; e-mail: meshkov@jinr.ru
}

on during the next years and a special transportation channel of monochromatic positrons and experimental stations for the PAS application equipped with instrumentation for spectroscopy was constructed. The first version of PAS-so called Doppler spectroscopy-was developed and used for performing about 30 experiment runs per year to study different kinds of materials $[2,3]$ :

1. metals and alloys: steel embrittlement in the reactor pressure vessel, fatigue effects in aircraft wings and different power units (screw propellers), corrosion of metallic structures;

2. ultra fine-grained metals-studies of structure;

3. semiconductors: studies of atomic defects in semiconductors (independent of its doping and conductivity).

(Polymers and different plastic materials are not allowed due to vacuum restriction).

The most effective version of PAS-Positron Annihilation Lifetime Spectroscopy (PALS) is being developmed presently. PALS has a specific sensitivity to vacancy-type defects which makes their identification straightforward and can be applied to any material [4]. The PALS version is developed at LEPTA. It is based on the original scheme of formation of an ordered positron flux proposed by the authors. A special beam transfer channel has been designed and commissioned this year.

The facility has a possibility of application of slow monochromatic positron beam with variable and wellcontrolled positron energy. 


\section{Generation of slow monochromatic positron flux}

In laboratory studies (non-accelerator experiments), the most common source is based on the positronactive isotope ${ }^{22} \mathrm{Na}$. Its positrons have a very wide energy spectrum with the highest energy of $545 \mathrm{keV}$ (Fig. 1a). The Cryogenic Source of Slow Monochromatic Positrons (CSSMP) at LEPTA has a different design $[5,6]$. The CSSMP is cooled down with liquid helium (LHe) to $7 \mathrm{~K}$ on the ${ }^{22} \mathrm{Na}$ isotope tablet. The tablet and the copper cone that envelopes the capsule with the isotope tablet are subjected to the directed flow of gaseous neon. It condenses to the solid state and emits positrons slowing down when crossing the layer of frozen neon. As a result, about $1 \%$ of full flux of positrons at the source exit has energy spectrum with the maximum about $2 \mathrm{eV}$ and the FWHM of $1.5-3 \mathrm{eV}$ (Fig. 1b). The source is suspended at the positive potential about $50 \mathrm{~V}$ and immersed into a solenoidal magnetic field that transports positrons to the target (the investigated sample).
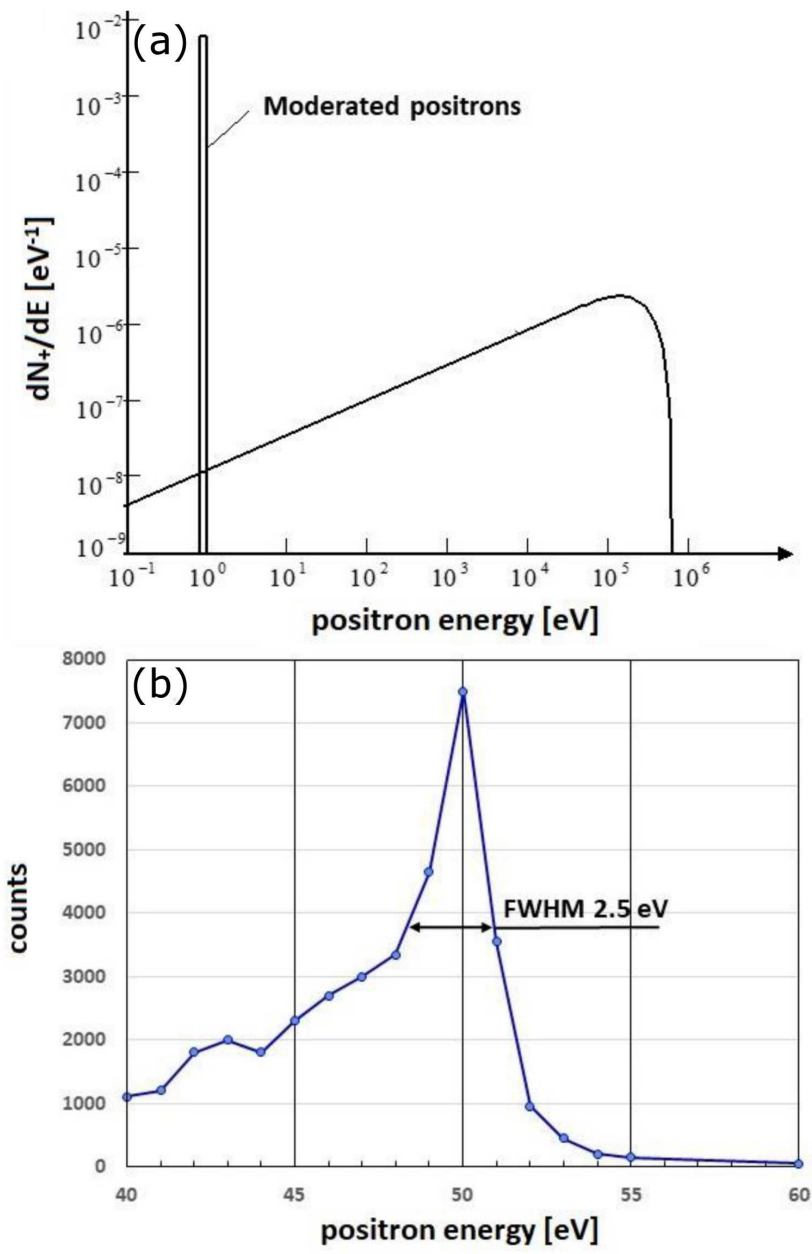

Fig. 1. (a) Theoretical spectrum of ${ }^{22} \mathrm{Na}$ isotope [1] and (b) experimental spectrum of moderated positrons [2].
To separate positrons of low and high energy, the section of the channel with the additional transverse field is used. This allows to obtain the monochromatic positron flux at the target.

\section{Studies using DBAL PAS method at LEPTA facility}

During the years 2014-2018 about 80 runs for PAS were performed at the LEPTA facility. The samples of irradiated metals [7-11] as well as subjected to a surface treatment $[12,13]$ were subsequently investigated by the DBAL PAS method.

Simultaneously the PALS method was used with the conventional ${ }^{22} \mathrm{Na}$ positron source of wide energy spectrum [14]. This enabled investigations of the samples of organic materials which are not compatible with the high vacuum in the LEPTA chamber.

During the years 2014-2016 important steps in the development of the PAS instrumentation was performed at LEPTA. First, a new ${ }^{22} \mathrm{Na}$ positron source was purchased from the iThemba Labs based on the long standing collaboration between our Institutions. The old source lost its radioactivity largerly from the fabrication time in 2006 (2.7 years of half-life). At the same time helium cryocooler was purchased and a new case of the CSSMP was designed and fabricated.

In November 2016 a new Cryogenic Source CSSMP-2 was mounted in the LEPTA injector. The new source has increased the research efficiency and operability significantly (Table I).

Parameters of two CSSMP versions

TABLE I

\begin{tabular}{l|c|c}
\hline \hline \multicolumn{1}{c|}{ Parameter } & CSSMP-1 & CSSMP-2 \\
\hline cooling & $\begin{array}{c}\text { hand assembling } \\
\text { with LHe Dewar }\end{array}$ & $\begin{array}{c}\text { closed cycle } \\
\text { with cryocooler }\end{array}$ \\
\hline preparation time & $\approx 7 \mathrm{~h}$ & $\approx 3 \mathrm{~h}$ \\
\hline supplying of LHe & hard problem & no \\
\hline flux of slow positrons & $1.5 \times 10^{6} \mathrm{~s}^{-1}$ & $3.3 \times 10^{6} \mathrm{~s}^{-1}$
\end{tabular}

\section{Towards the PALS method at LEPTA}

\author{
4.1. Principle of formation \\ of the ordered positron flux (OPF)
}

The basic idea of the OPF formation is subjecting the flux of monochromatic positrons with a periodic pulsed accelerating (decelerating) voltage of special form [4]. The voltage is a function of time of positron crossing the gap with applied voltage.

The principle of such a method is well known [15]. However, it is used yet with the harmonic RF voltage $[16,17]$.

In our scheme [14] the positrons for the CSSMP follow one another in a stream with an average periodicity of the order of 1 microsecond (intensity of the CSSMP). When crossing the gap with the RF ordering voltage 
the positron accelerates depending on time of arrival to the entrance of the RF gap. Then positrons reach the target strictly periodically, independently of time when they come to the RF gap. Thus, the random character of positron generation in the source is compensated-this is the ordering.

The positron transfer channel is equipped with an additional gap ("acceleration gap") with a static accelerating voltage of the order of $5 \mathrm{kV}$ that is placed after the ordering gap. This allows minimizing the influence of the positron energy spread existing at injection from the CSSMP.

Each pulse of the periodic ordering voltage is described with the following function [14]:

$$
F_{U}(x)= \begin{cases}U_{R F}^{(1)}(x), & 0 \leq x \leq 1 \\ U_{R F}^{(2)}(1) \frac{1.1-x}{0.1}, & 1<x \leq 1.1\end{cases}
$$

where

$$
\begin{aligned}
& U_{R F}^{(1)}\left(t_{\mathrm{inj}}\right)=\left[\left(1-\sqrt{\frac{2 E_{0}}{m}} \frac{t_{\mathrm{inj}}+\delta t_{2}}{L_{A}}\right)^{-2}-1\right] \frac{E_{0}}{e}, \\
& \delta t_{2}=L_{t}\left(\frac{1}{v_{a}\left(t_{\mathrm{inj}}\right)}-\frac{1}{v_{A}(0)}\right) .
\end{aligned}
$$

The second line in the formula for $F_{U}(x)$ describes the rear front of the pulse. Here, $x=t_{\text {inj }} / T_{0}$, $t_{\text {inj }}$ is the positron time injection into the ordering gap, and $T_{0}$ is the period of the ordering voltage. $E_{0} \sim 100 \mathrm{eV}$ is the positron energy at the exit of the CSSMP, $e$ and $m$ are the positron charge and mass, respectively. $L_{A}$ is the distance between the ordering and acceleration gaps, $v_{A}$ is the velocity at the exit of the acceleration gap of the positron injected at $t=0$ and $t_{\mathrm{inj}} . L_{t}$ is the distance between the acceleration gap and the target (the sample to be studied). The size (length) of both gaps can be so small that their influence on duration of positron travelling to the target is negligible.

\subsection{Synthesis of $U_{R F}(t)$ pulses with harmonic voltage}

The most convenient and simple way is forming the function of the periodic voltage pulses $F_{U}(x)$ by the harmonic functions which are the Fourier components of this function. The corresponding results of the Fourier transformation of the periodic function $F_{U}(x)$ are shown in Table II for the parameters chosen for the PALS channel at LEPTA [14].

Parameters of RF harmonics

TABLE II

\begin{tabular}{c|c|c|c|c}
\hline \hline$m$ & $A_{m}[\mathrm{~V}]$ & $\varphi_{m}[\mathrm{rad}]$ & $\lambda[\mathrm{m}]$ & $f[\mathrm{MHz}]$ \\
\hline 0 & 213.76 & - & - & - \\
1 & 85.44 & $\pi+0.265$ & 3.0 & 100 \\
2 & 36.54 & $\pi+0.667$ & 1.5 & 200 \\
3 & 21.43 & $\pi+0.978$ & 1.0 & 300 \\
4 & 13.93 & $\pi+1.281$ & 0.75 & 400
\end{tabular}

As indicated by the numerical simulation of the formation process, satisfactory results can be obtained using three harmonics $m=1,2,3$, and permanent voltage (positron energy shift) $m=0$.

\subsection{Synchronization and start-stop signals for PALS OPF}

The system of start-stop signals differs significantly from that used in the classical scheme: the signal from the decay gamma-quant of ${ }^{22} \mathrm{Na}$ does not correlate actually with positron travelling in the PALS channel. Therefore we suggest using a "timer" signal connected to a certain phase of RF voltage $U_{R F}(t)$, for instance the zero phase ("synchro-signal", Fig. 2). Then the signal of a photon from the positron annihilation gives the start signal and the next synchro-signal, nearest to the start one, the stop signal. Then, to find the positron lifetime, there must be known the value of its $t_{\text {flight }}$-the time duration of positron travelling from the entrance of the ordering gap to the target. The value of $t_{\text {flight }}$ is the same for all positrons. Therefore one can find $t_{\text {flight }}$ using the reference sample whose lifetime is measured for instance, using the conventional PALS set up (Sect. 2). Thus

$$
\begin{aligned}
& t_{\text {flight }}=\left(t_{\text {start }}-\tau_{\text {life }}\right)_{\text {reference }} \\
& \text { and }\left(\tau_{\text {life }}\right)_{\text {sample }}=t_{\text {stop }}-t_{\text {start }}-t_{\text {flight }} .
\end{aligned}
$$

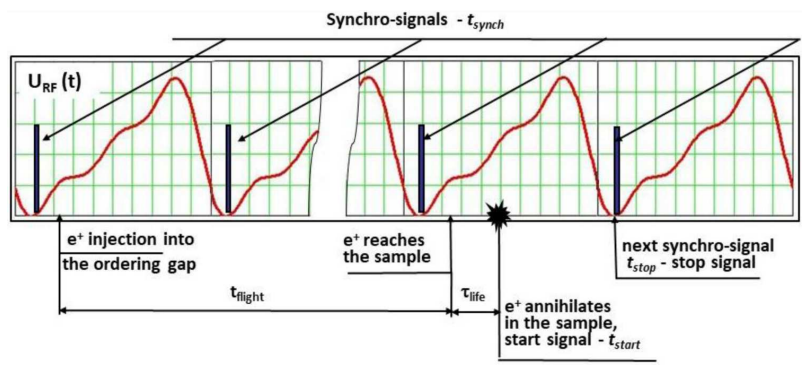

Fig. 2. Scheme of forming start-stop signals (description in the text).

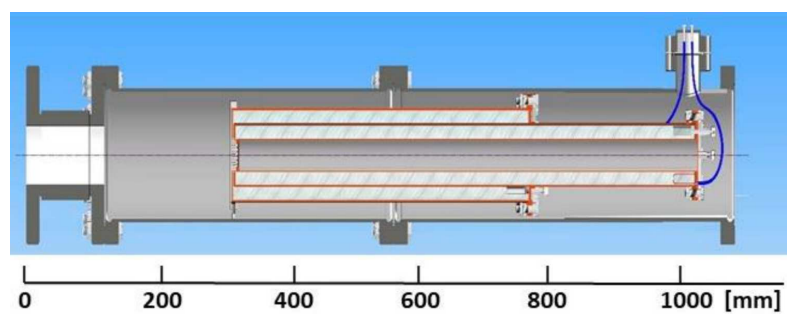

Fig. 3. Drawing of a three-frequency quarter-wave resonator.

\subsection{Hardware for the synthesis} of three harmonics for OPF formation

The ordering gap is placed at the end face of two coaxial cylindrical cavities which generate three first harmonics of RF voltage: $\lambda_{1}$ and $\lambda_{3}$ harmonics are excited 
in the common cavity of the length of $\lambda_{1} / 4$, the $\lambda_{2}$ harmonics is excited in the other cavity of the length $\lambda_{1} / 8$ (Fig. 3). Three grids of high transparency cover the end face of the cavities. This RF system is designed and fabricated currently.

\section{Conclusions}

1. The proposed method of the ordered positron flux formation is an advanced version of the well known method of bunching continuous particle flux with a system of harmonic RF voltage. The method promises a significant increase of ordering efficiency and higher precision of positron lifetime measurement - below 30 ps.

2. Design and construction of the PALS OPF system is in progress at the LEPTA facility at JINR and it will provide a new quality of research with PAS in solid matter physics.

\section{References}

[1] R. Krause-Rehberg, S.H. Leipner, Positron Annihilation in Semiconductors, Springer, Berlin, 1998.

[2] P. Horodek, M. Bugdol, A. Kobets, I. Meshkov, O. Orlov, A. Rudakov, A. A. Sidorin, S. Yakovenko, Phys Part. Nucl. 11, 708 (2014).

[3] M.K. Eseev, A.A. Goshev, P. Horodek, S.N. Kapustin, A.G. Kobets, C.S. Osokin, Nanosys.: Phys. Chem. Math. 7, 180 (2016).
[4] F. Tuomisto, I. Makkonen, Rev. Mod. Phys. 85, 1583 (2013).

[5] V. Bykovsky, M. Eseev, I. Meshkov, V. Pavlov, R. Pivin, A. Rudakov, G. Trubnikov, S. Yakovenko, in: Proc. RuPAC, Development Of The Positron Injector For Lepta Facility, Zvenigorod (Russia) 2008.

[6] P. Horodek, A. Kobets, I. Meshkov, A. A. Sidorin, O. Orlov, Nukleonika 60, 725 (2015).

[7] P. Horodek, J. Dryzek, V.A. Skuratov, Vacuum 138, 15 (2017).

[8] J. Dryzek, P. Horodek, V.A. Skuratov, Acta Phys. Pol. A 132, 1585 (2017).

[9] P. Horodek, J. Dryzek, V.A. Skuratov, Rad. Phys. Chem. 122, 60 (2016).

[10] P. Horodek, V.A. Skuratov, Surf. Coat. Technol. 296, 65 (2016).

[11] J. Dryzek, P. Horodek, Appl. Phys. A 121, 289 (2015).

[12] P. Horodek, K. Siemek, J. Dryzek, A.G. Kobets, M. Wrobel, Tribol. Lett. 65, (2017).

[13] P. Horodek, K. Siemek, A.G. Kobets, I.N. Meshkov, Appl. Surf. Sci. 333, 96 (2015).

[14] I. Meshkov, Phys. Part. Nucl. Lett. 15, 790 (2018).

[15] P. Coleman, Positron Beams and Their Applications, World Scientific, Singapore 2000.

[16] R. Suzuki, Y. Kobayashi, T. Mikado, H. Ohgaki, M. Chiwaki, T. Yamazaki, Hyperfine Interact. $\mathbf{8 4}$, 345 (1994).

[17] B.E. O'Rourke, Def. Diff. Forum 331, 75 (2012). 